\title{
CCD-based imaging and 3D space-time mapping of terahertz fields via Kerr frequency conversion
}

\author{
Matteo Clerici, ${ }^{1,2 * *}$ Daniele Faccio, ${ }^{2}$ Lucia Caspani, ${ }^{1}$ Marco Peccianti, ${ }^{3}$ Eleonora Rubino, ${ }^{4}$ \\ Luca Razzari, ${ }^{1}$ François Légaré, ${ }^{1}$ Tsuneyuki Ozaki, ${ }^{1}$ and Roberto Morandotti $^{1}$ \\ ${ }^{1}$ INRS-EMT, 1650 Blvd. Lionel-Boulet, Varennes, Québec J3X 1S2, Canada \\ ${ }^{2}$ School of Engineering and Physical Sciences, Heriot-Watt University, SUPA, Edinburgh EH14 4AS, UK \\ ${ }^{3}$ Institute for Complex Systems (ISC), CNR, via dei Taurini 19, Rome 00185, Italy \\ ${ }^{4}$ Dipartimento di Scienza e Alta Tecnologia, Università degli Studi dell’Insubria, via Valleggio 11, Como 22100, Italy \\ ${ }^{*}$ Corresponding author: clerici@emt.inrs.ca
}

\begin{abstract}
We investigate the spatially and temporally resolved four-wave mixing of terahertz (THz) fields and optical pulses in large-bandgap dielectrics, such as diamond. We show that it is possible to perform beam profiling and space-time resolved mapping of $\mathrm{THz}$ fields by encoding the spatial information into an optical signal, which can then be recorded by a standard CCD camera.
\end{abstract}

Since the seminal work of Hu and Nuss [1] ], time-resolved imaging of terahertz (THz) radiation (T-ray imaging) has become a widely investigated and employed technique. The initial efforts carried out in the 1990s (see, e.g., [1-4] and references therein) triggered the interest of the optics community, and a large literature followed (see, e.g., $[\underline{5}, \underline{6}]$ for a review). Currently, T-ray imaging systems are commercially available and widely employed for a diverse range of applications. For several applications, however, spectroscopic information is not required and hence imaging techniques relying on the analysis of the spatially resolved electro-optical effect in second-order crystals may result in unnecessary efforts. For such cases, $\mathrm{THz}$ focal plane detectors are the most straightforward tools. Although research on $\mathrm{THz}$ focal plane arrays is proceeding very rapidly (see, e.g., $[\underline{7,8]}$ ), such devices are still not readily available or as efficient as CCD and CMOS cameras for the visible part of the spectrum and are typically quite expensive.

Alternatively, the detection of $\mathrm{THz}$ fields can also be performed via the $\mathrm{THz}$ electric-field-induced secondharmonic generation, or EFISH (or TFISH, when considering the THz electric field), in Kerr media. Such an effect is a degenerate case of four-wave mixing (FWM) between an unknown $\mathrm{THz}$ and an optical probe field, which upshifts the frequency of the $\mathrm{THz}$ pulse into the visible, where it could be easily recorded with standard silicon detectors as a function of the delay with the probe pulse. This effect has been employed, for instance, for the detection of freely propagating millimeter waves and $\mathrm{THz}$ pulses $[9,10]$. Furthermore, TFISH in gas media is the key for the coherent detection of ultra-broadband $\mathrm{THz}$ pulses $[\underline{11}, \underline{12}]$.

In this Letter, we show that spatially resolved FWM between $\mathrm{THz}$ fields and a near-infrared probe in largebandgap (solid state) Kerr media can be employed for $\mathrm{THz}$ beam profiling and imaging via standard CCD cameras. We also show an example of a space-time resolved measurement, namely, a three-dimensional (3D) tomographic reconstruction of the $\mathrm{THz}$ pulse, or 3Dmapping [13].

In our experiments, we generate broadband $\mathrm{THz}$ pulses by focusing in air an intense (ionizing) optical field and its second harmonic, generated via a beta barium borate crystal (see Fig. 1) [14-16]. The generated THz pulse is then collimated by a $101.6 \mathrm{~mm}$ equivalent focal length (EFL) gold-coated parabolic mirror, and the pump pulse is removed by two low-pass multimesh filters (QMC Instruments, UK) with $20 \mathrm{THz}$ cut-in frequency and nearly $40 \mathrm{~dB}$ rejection for $\nu>20 \mathrm{THz}$. The $\mathrm{THz}$ beam is then refocused by means of a $50.8 \mathrm{~mm}$ EFL mirror to an aperture-limited spot size. A second pair of parabolic mirrors (50.8 mm EFL) forms an $M=1$ telescope that images the focused beam (at the image plane in Fig. 1) into the detection plane. A detailed description of the $\mathrm{THz}$ source and the characterization of the generated THz field can be found in [16] while in the inset of Fig. 1 we show the $\mathrm{THz}$ electric field and spectrum recorded via air-biased coherent detection (ABCD) [12].

In order to prove the $\mathrm{THz}$ imaging capabilities of FWM we employed a $500 \mu \mathrm{m}$ thick, $4 \mathrm{~mm} \times 4 \mathrm{~mm}$ aperture, $\langle 100\rangle$-cut, single crystal diamond sample as the nonlinear medium, and we overlapped the THz field with a collimated, $795 \mathrm{~nm}, 60 \mathrm{fs}$ duration probe pulse passing through a $1.5 \mathrm{~mm}$ diameter hole in the last parabolic mirror of the THz telescope (see Fig. 1). The choice of diamond as Kerr medium is due to its remarkable transparency properties (absorption $<1 \mathrm{~cm}^{-1}$ ) in both the infrared and $\mathrm{THz}$ spectral regions. When the $\mathrm{THz}$ and the probe beams overlap, wave-mixing occurs and a signal proportional to the $\mathrm{THz}$ intensity is generated at a wavelength close to the probe second harmonic $(400 \mathrm{~nm}$, for low conversion efficiencies $I_{\mathrm{FWM}} \propto I_{\text {probe }}^{2} I_{\mathrm{THz}}$ ).

We removed the probe fundamental harmonic with a suitable bandpass filter, and we imaged the diamond surface onto a cooled CCD camera (620i, QSI). In Fig. 2(a) we show the recorded image, acquired by averaging over 100 laser shots and for $350 \mu \mathrm{J}$ probe pulses energy. The 


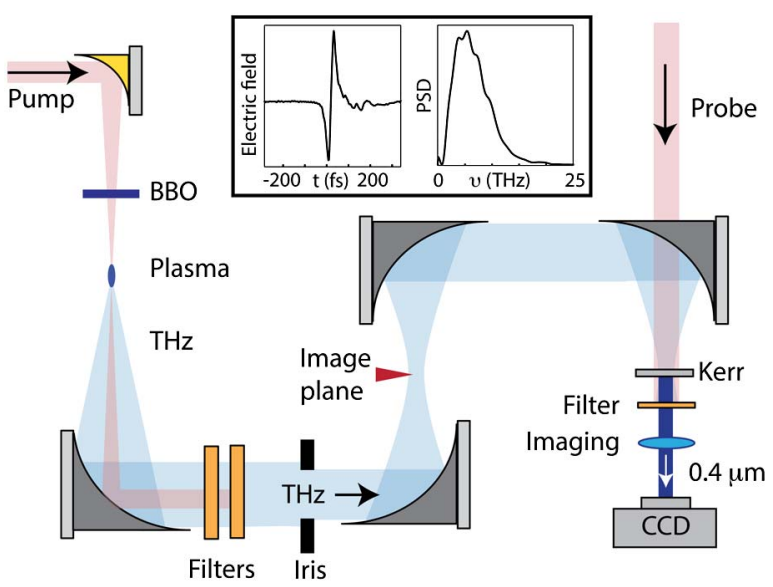

Fig. 1. Sketch of the experimental setup employed for the generation and imaging of $\mathrm{THz}$ pulses. In the inset, we show the time resolved electric field profile and the spectrum recorded via $\mathrm{ABCD}$ detection (see text).

horizontal and vertical axes are corrected in order to account for the magnification of the last imaging system. From both the imaging geometry and further optical calibrations we estimate a magnification factor $M=3.9$.

The image clearly shows that the THz beam profile is Bessel-shaped rather than Gaussian. In Fig. 2(b) we show the beam profile along the $y$ axis extracted from the image at $x=0$ (red, dashed curve), overlapped with a fit considering a Bessel beam apodized with a Gaussian envelope, namely a Bessel-Gauss beam (green, shaded curve):

$$
I(y)=J_{0}^{2}(\alpha y) \exp \left[-\left(y / \sigma_{y}\right)^{2}\right],
$$

where $\alpha \simeq 0.0412 \pm 0.0002 \mu \mathrm{m}^{-1}$ and $\sigma_{y}=76 \pm 2 \mu \mathrm{m}$ (we considered here the standard deviations resulting from the nonlinear fit). In order to compare the results obtained with the proposed nonlinear imaging technique with what was obtained by traditional means, we recorded the THz beam profile in the image plane both with a camera (PV 320, Electrophysics; see [16]) and with a knife-edge measurement, moving a blade in the image plane and recording the signal in the detection plane with

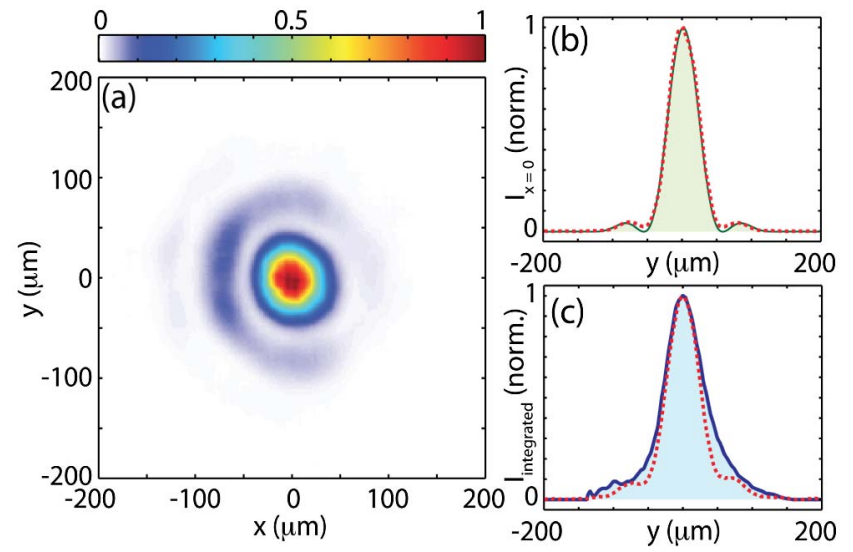

Fig. 2. (a) THz beam profile recorded via the proposed nonlinear imaging technique. (b) Section of the THz beam along the $y$ coordinate $(x=0)$ (red, dashed curve) overlapped with a Bessel-Gauss beam fit (green, shaded). (c) Measured profile integrated on the $x$ direction (red, dashed) compared to the result of a knife-edge measurement (blue, shaded). a pyroelectric detector. The beam profile recorded with the camera and fitted by a Gaussian function resulted in $\sigma_{G} \simeq 85.5 \mu \mathrm{m}$, compatible with the width of the Gaussian apodization recorded via the nonlinear imaging technique. We note that the large pixel size of the PV 320 camera $(48.5 \mu \mathrm{m})$ prevents recording any fine detail, such as the Bessel structure. The $\mathrm{THz}$ beam profile retrieved via the differentiation of the knife-edge data (considering an $x-y$ factorable profile) is compared in Fig. 2(c) (blue, shaded curve) with the beam profile recorded via the proposed nonlinear technique and integrated along the $x$ direction (red, dashed curve), showing a good agreement between the two techniques. It is worth noting that since the beam-profile resolution of the proposed nonlinear technique is limited by the numerical aperture of the imaging system operating on the visible signal, under proper conditions subwavelength resolution may be achieved for the $\mathrm{THz}$ radiation.

To further prove that the Bessel profile recorded via the nonlinear technique is not an artifact, we imaged the beam profile with the $\mathrm{THz}$ camera for different planes along the $z$ (propagation) coordinate, around the image plane (data not shown). As expected for a two-color plasma source (see, e.g., [17]) we clearly observed that the $\mathrm{THz}$ emission is conical, with a carrier cone-angle of $\theta=12.8 \pm 0.4 \mathrm{deg}$ (we considered the standard deviations resulting from the linear fit). This angle is indeed in agreement with the $\alpha$ value used in our fit (Eq. 1) assuming a carrier frequency of $\simeq 9 \mathrm{THz}$.

To prove that the imaging capabilities of the proposed technique extend beyond simple beam profiling, we have imaged an opaque object placed in the image plane, namely a $125 \mu \mathrm{m}$ diameter single-mode silica fiber. To this purpose we have increased the $\mathrm{THz}$ spot size by closing an iris in the collimated THz beam path (as shown in Fig. 1). In Figs. 3(a) and 3(b) we show the recorded visible signal without and with the fiber, respectively. As expected, Fig. 3(b) clearly shows a dark area of nearly $130 \mu \mathrm{m}$ width, induced by the fiber.

Curiously, we noted that although the $\mathrm{THz}$ and the probe pulse have 90 and 60 fs duration, respectively, the recorded signal is insensitive to the two-pulse delay over an interval of nearly 8 ps, as shown in Fig. 4(a). The origin of the wavelength selectivity of the proposed nonlinear imaging system and of its insensitivity to the pulse-to-pulse delay can be found in the phase-matching properties of the FWM process employed for the $\mathrm{THz}$
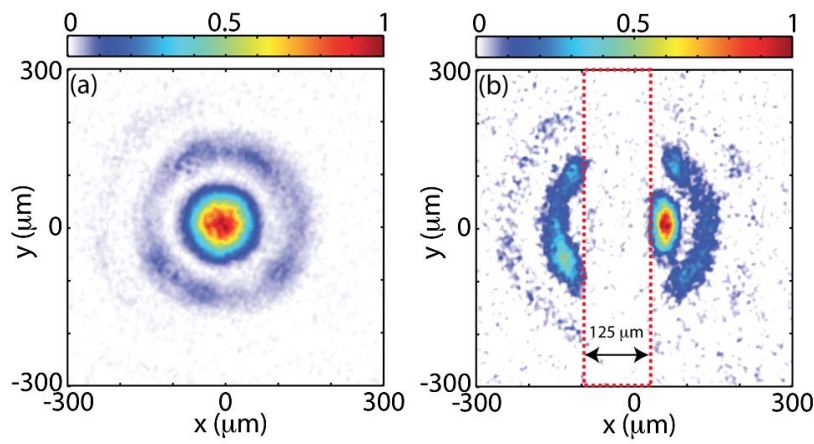

Fig. 3. (a) THz beam profile recorded with a closed iris in the THz beam-path. (b) Same as (a) with a single mode fiber blocking the $\mathrm{THz}$ beam in the image plane. 
(a)

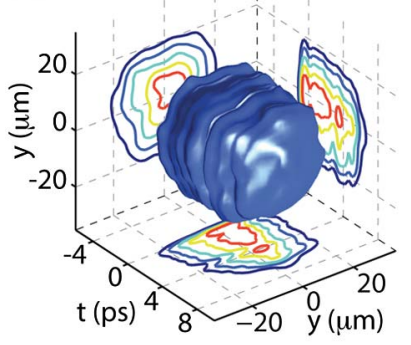

(b)

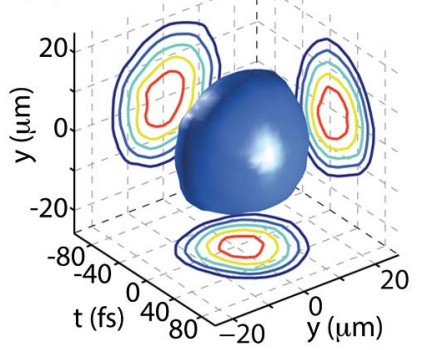

Fig. 4. (a) and (b) are 3D mapping of the THz pulse performed via the Kerr nonlinearity in diamond and borosilicate glass microscope coverslip, respectively. The figures show an isoamplitude surface at $50 \%$ of the maximum. The curves on the sides are iso-amplitude levels for the corresponding projections (50\%-90\%).

up-frequency shifting. Indeed it has been recently demonstrated that the FWM between broadband $\mathrm{THz}$ and $\simeq 800 \mathrm{~nm}$ pulses in diamond has a component of difference frequency generation that is naturally phase matched at $\simeq 10 \mathrm{THz}$ for fields counterpropagating to the optical pulse [18], in agreement with the $9 \mathrm{THz}$ carrier frequency inferred from the above-mentioned Bessel fit. Furthermore, for a $500 \mu \mathrm{m}$ thick diamond sample the counterpropagating wave-mixing signal is indeed expected to extend nearly $8 \mathrm{ps}$ in the delay coordinate (see [18]). The image is hence generated by both the forward and, for longer delays, the $\mathrm{THz}$ component reflected from the diamond-air interface (as shown in [18], this is the strongest component resulting from the $\mathrm{FWM}$ in diamond). On the one hand, this implies that time-resolved measurements cannot be performed with a parallel-plate diamond sample. On the other hand, however, the backward interaction allows direct measuring of the time-integrated beam profile with short probe pulses without scanning.

In order to perform space- and time-resolved $\mathrm{THz}$ characterization, it is essential to remove the backward phase-matched component (due to the fact that it is delayinsensitive), which took place in all of the transparent materials that we investigated ( $\mathrm{KBr}, \mathrm{CsI}, \mathrm{Al}_{2} \mathrm{O}_{3}$, and others). An alternative to employing wedge crystals is that of relying on THz-absorptive Kerr media. The THz radiation in this case is absorbed before reaching the output facet of the nonlinear medium, where the reflection occurs in the case of transparent samples. Thus the interaction occurs only in the copropagating geometry, and the measurement is sensitive to the THz-probe delay. A 3D mapping of the $\mathrm{THz}$ pulse can indeed be reconstructed employing a borosilicate glass microscope coverslip, as shown in Fig. 4(b). In this experiments the temporal duration recorded via the $3 \mathrm{D}$ mapping $(\simeq 110 \mathrm{fs})$ is compatible with the duration of the THz pulse obtained by applying a Hilbert transform to the electric field trace recorded via $\mathrm{ABCD}(\simeq 90$ fs; see inset in Fig. 1) [1ㅡ], once a suitable absorption spectrum at THz frequencies is considered for the borosilicate glass. From the spatial point of view, the small beam width measured with the borosilicate glass also indicates that the mixing process converts mainly the higher-frequency components of the THz pulse.

In conclusion, we have shown that imaging at $\mathrm{THz}$ frequencies can be performed with a standard CCD

camera exploiting THz-frequency upconversion in transparent Kerr media. The counterpropagating phasematched interaction occurring at $\mathrm{THz}$ frequencies allows for a delay-insensitive measurement, rendering the described effect of interest as a possible alternative to focal-plane arrays operating at $\mathrm{THz}$ frequencies. We have also shown that space-time resolved $\mathrm{THz}$ mapping could be performed in absorbing Kerr media.

The research in Canada has been funded by NSERC (Natural Sciences and Engineering Research Council of Canada), FQRNT (Le Fonds québécois de la recherche sur la nature et les technologies), and MDEIE (Ministère du Développement économique, de l'Innovation et de l'Exportation). M. C. acknowledges the support from the People Programme (Marie Curie Actions) of the European Union's Seventh Framework Programme (FP7/2007-2013), REA GA 299522.; D. F. acknowledges financial support from the Engineering and Physical Sciences Research Council (EPSRC), Grant EP/J00443X/1, and from the European Research Council under the European Union's Seventh Framework Programme (FP7/2007-2013)/ERC GA 306559. L. C. and E. R. acknowledge support from FQRNT. The authors acknowledge help from the ALLS staff, B. E. Schmidt, N. Thiré, M. Cassataro, and M. Shalaby and support from Gentec-EO.

\section{References}

1. B. B. Hu and M. C. Nuss, Opt. Lett. 20, 1716 (1995).

2. D. M. Mittleman, R. H. Jacobsen, and M. C. Nuss, IEEE J. Sel. Topics. Quantum Electron. 2, 679 (1996).

3. Q. Wu, T. D. Hewitt, and X.-C. Zhang, Appl. Phys. Lett. 69, 1026 (1996).

4. Z. Jiang and X. Zhang, IEEE Trans. Microwave Theory Tech. 47, 2644 (1999).

5. W. L. Chan, J. Deibel, and D. M. Mittleman, Rep. Prog. Phys. 70, 1325 (2007).

6. P. U. Jepsen, D. G. Cooke, and M. Koch, Laser Photonics Rev. 5, 124 (2011).

7. A. W. M. Lee and Q. Hu, Opt. Lett. 30, 2563 (2005).

8. A. Rogalski and F. Sizov, Opto-Electron. Rev. 19, 346 (2011).

9. C. Ohlhoff, C. Meyer, G. Lüpke, T. Löffler, T. Pfeifer, H. G. Roskos, and H. Kurz, Appl. Phys. Lett. 68, 1699 (1996).

10. A. Nahata and T. F. Heinz, Opt. Lett. 23, 67 (1998).

11. J. Dai, X. Xie, and X.-C. Zhang, Phys. Rev. Lett. 97, 103903 (2006).

12. N. Karpowicz, J. Dai, X. Lu, Y. Chen, M. Yamaguchi, H. Zhao, X.-C. Zhang, L. Zhang, C. Zhang, M. Price-Gallagher, C. Fletcher, O. Mamer, A. Lesimple, and K. Johnson, Appl. Phys. Lett. 92, 011131 (2008).

13. M. A. C. Potenza, S. Minardi, J. Trull, G. Blasi, D. Salerno, A. Varanavičius, A. Piskarskas, and P. Di Trapani, Opt. Commun. 229, 381 (2004).

14. D. J. Cook and R. M. Hochstrasser, Opt. Lett. 25, 1210 (2000).

15. K.-Y. Kim, J. H. Glownia, A. J. Taylor, and G. Rodriguez, Opt. Express 15, 4577 (2007).

16. M. Clerici, M. Peccianti, B. E. Schmidt, L. Caspani, M. Shalaby, M. Giguère, A. Lotti, A. Couairon, F. Légaré, T. Ozaki, D. Faccio, and R. Morandotti, "Scaling mechanism for efficient wavelength conversion in laser plasmas," arXiv 1207.4754 (2012).

17. H. Zhong, N. Karpowicz, and X.-C. Zhang, Appl. Phys. Lett. 88, 261103 (2006).

18. M. Clerici, L. Caspani, E. Rubino, M. Peccianti, M. Cassataro, A. Busacca, T. Ozaki, D. Faccio, and R. Morandotti, Opt. Lett. 38, 178 (2013). 